\title{
EPIDEMIOLOGY
}

\section{Infective endocarditis rises as prophylactic antibiotic use falls}

Since the UK National Institute for Health and Care Excellence (NICE) modified its guidelines in 2008 and recommended the cessation of prophylactic antibiotic prescription to prevent infective endocarditis, the incidence of this disease has significantly increased above the historical trend. These data, presented at the AHA Scientific Sessions 2014 in Chicago, IL, USA and published in The Lancet, highlight a trend that should be further investigated.

The investigators performed a retrospective, secular trend study with prescription data and anonymized discharge diagnoses from patients treated in the UK National Health Service. Using this resource, the team identified that antibiotic prescriptions to prevent infective endocarditis dropped from a mean of 10,900 per month in the period from 1 January 2004 to 31 March 2008 (when the updated NICE guidelines were introduced) to a mean of 2,236 per month from 1 April 2008 to 31 March $2013(P<0.0001)$. During the final 6 months of the study, the mean number of prescriptions fell further to only 1,307 per month. The team also identified that between 1 January 2000 and 31 March 2013, 19,804 patients were given a primary discharge diagnosis of acute or subacute infective endocarditis.

Although an upward trend in the incidence of infective endocarditis was observed in the data from 1 January 2000 to 31 March 2008, the trend in the incidence of infective endocarditis increased significantly after the introduction of the NICE guidelines. This increase was calculated as 0.11 cases per 10 million individuals per month $(95 \% \mathrm{CI}$ $0.05-0.16, P<0.0001)$. The team also estimated that by 31 March 2013, 34.9 (95\% CI 7.9-61.9) more cases of infective endocarditis had occurred above the historical trend than if the updated NICE guidelines had not been introduced. However, the investigators did not identify a significant increase in mortality owing to infective endocarditis after 31 March 2008 (change in slope $0.01 ; 95 \%$ CI -0.01 to 0.02 , $P=0.394$ ). The trends in incidence and mortality were observed in both the population-corrected and population-uncorrected data, and in individuals who were classified as at high-risk and lower-risk of infective endocarditis.

The investigators conclude that "although we have identified a temporal relation between these two changes [prophylactic antibiotic use and incidence of infective endocarditis], our data do not establish a causal link". In an associated commentary, Xavier Duval and Bruno Hoen highlight that "although the cessation of antibiotic prophylaxis was recommended because little evidence existed to support efficacy, the present data [by Dayer et al.] do not provide very strong evidence either". Duval and Hoen also suggest that "experts stop elaborating guidelines for infective endocarditis prophylaxis, and urgently join forces to mount an international collaboration to do the appropriate clinical trials".

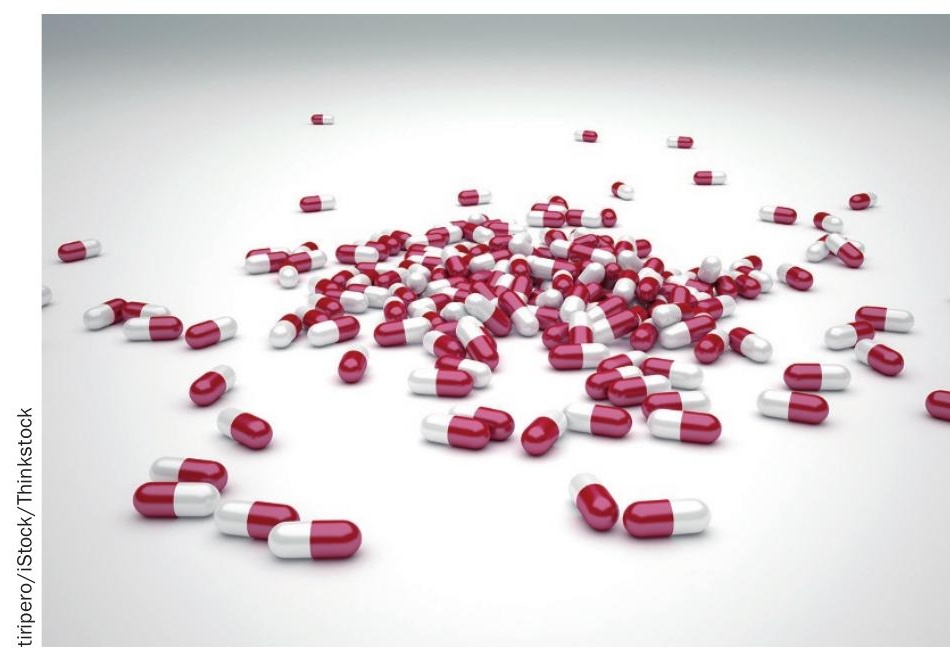

